\title{
Printable luminescent down shifter for enhancing efficiency and stability of organic photovoltaics
}

Kettle, J.; Bristow, N.; Gethin, D.T.; Tehrani, Z.; Moudam, O.; Li, B.; Katz, E.A.; Benatto, Gisele Alves dos Reis; Krebs, Frederik C

Published in:

Solar Energy Materials \& Solar Cells

Link to article, DOI:

10.1016/j.solmat.2015.09.037

Publication date:

2016

Document Version

Peer reviewed version

Link back to DTU Orbit

Citation (APA):

Kettle, J., Bristow, N., Gethin, D. T., Tehrani, Z., Moudam, O., Li, B., Katz, E. A., Benatto, G. A. D. R., \& Krebs, F. C. (2016). Printable luminescent down shifter for enhancing efficiency and stability of organic photovoltaics. Solar Energy Materials \& Solar Cells, 144, 481-487. https://doi.org/10.1016/j.solmat.2015.09.037

\section{General rights}

Copyright and moral rights for the publications made accessible in the public portal are retained by the authors and/or other copyright owners and it is a condition of accessing publications that users recognise and abide by the legal requirements associated with these rights.

- Users may download and print one copy of any publication from the public portal for the purpose of private study or research.

- You may not further distribute the material or use it for any profit-making activity or commercial gain

- You may freely distribute the URL identifying the publication in the public portal 
Printable Luminescent Down Shifter for Enhancing Efficiency and Stability of Organic Photovoltaics

\author{
J. Kettle*1, N. Bristow ${ }^{1}$, D.T. Gethin ${ }^{2}$, Z. Tehrani' ${ }^{2}$, O.Moudam ${ }^{1}$, B. Li ${ }^{3}$, E.A.Katz ${ }^{3,4}$, G.A. dos Reis \\ Benatto $^{5}$, F.C. Krebs ${ }^{5}$
}

1. School of Electronic Engineering, Bangor University, Dean St, Gwynedd, Bangor, LL57 1UT, Wales, UK *Contact details; Tel: +44 (0) 1248 382471; Fax: E-mail:j.kettle@bangor.ac.uk

2. Welsh Coating and Printing Centre (WCPC), Swansea University, Singleton park, Swansea, SA2 8PP, Wales, UK

3. Department of Solar Energy and Environmental Physics, Swiss Institute for Dryland Environmental and Energy Research, J. Blaustein Institutes for Desert Research, Ben-Gurion University of the Negev, Sede Boker Campus 84990, Israel

4. Ilse Katz Inst. of Nano-Science and Technology, Ben-Gurion University of the Negev, Be'er Sheva 84105, Israel

5. National Laboratory for Sustainable Energy, Technical University of Denmark, Frederiksborgvej 399, DK-4000 Roskilde, Denmark

\begin{abstract}
The proof of concept of using luminescent down shifting (LDS) layers as alternative UV filters for P3HT:PCBM OPVs is demonstrated using a lanthanide-based metal complex. The results are verified using a combination of indoor light soaking, with single cell devices, and outdoor performance monitoring, using a 16-cell monolithically connected OPV module. By applying the LDS layer, a 5\% relative enhancement in photocurrent is observed for both sets of devices. More significantly, indoor light soaking tests on single cell devices without encapsulation showed an $850 \%$ enhancement in the measured half-life $\left(T_{50 \%}\right)$. The OPV modules were encapsulated and tested for outdoor stability over a 70 day period in the Negev desert, Israel. The modules made with the LDS filter are shown to match the stability of those made with a commercial UV filter and outperform the modules with no filter applied, with a $51 \%$ enhancement in the measured stability $\left(T_{75 \%}\right)$. Significantly, the work provides clear experimental evidence that the LDS layer can act as a UV filter in OPVs without compromising the efficiency of the solar cell, thus providing an added benefit over commercial UV filters.
\end{abstract}

\title{
Keywords
}

Organic photovoltaics, degradation, outdoor performance, UV filter, luminescent material 


\section{Introduction}

Organic Photovoltaics (OPVs) based on solution processable polymers and fullerenes have attracted remarkable interest during the last decade because of their potential for low cost, printability and flexibility. Recent research in this area has led to the report of a power conversion efficiency (PCE) of over 10\% [1]. However, there are still challenges that the technologists developing OPVs need to overcome before it can become a mainstream solar technology, in particular combining high efficiency and long term operational stability in outdoor environments [2]. The causes of instability are numerous and complex and are induced or accelerated by a range of environmental effects such as temperature, water and ultraviolet (UV) light exposure [3], with the latter leading to photooxidation of active layer components or polymer chain scission [4,5]. As a result, the technology is unsuitable for outdoor applications unless a UV filter is applied. One major disadvantage of incorporating a UV filter into the module is that the application is normally accompanied by a reduction in efficiency, due to increased optical losses [6].

In recent years, luminescent down-shifting (LDS) materials have been widely studied and applied to photovoltaics to improve the PCE $[7,8]$. These materials absorb photons at wavelengths $(\lambda)$ where the PV responds poorly $(\lambda<400 \mathrm{~nm})$ and re-emit photons in the visible spectrum, where the spectral response of the PV is much greater. For this reason, the application of an LDS onto an OPV could actually serve two purposes: (1) to filter the incident UV light and to suppress OPV degradation and (2) to improve the response of the OPV at short-wavelength.

Whilst the potential for efficiency and stability enhancements via LDS layers have been reported in a wide range of PV technologies, including Dye Sensitised Solar Cells (DSSCs) [9], there are only a limited number of studies that have been carried out on OPV materials [10] and devices $[11,12,13]$. In particular, Sloff et al. and Engmann et al. have reported promising results that show a photocurrent improvement by applying LDS layers in OPVs, but the stability issue was not addressed. This paper reports the proof of concept of improvement of both the lifetime and efficiency of OPV cells by applying LDS layers. The LDS layer can replace conventional UV filters, which are known to reduce the solar cell performance after application. The results are verified using a combination of indoor light soaking and outdoor performance monitoring, which was conducted over a 70 day period in the Negev desert in Israel.

\section{Experimental}

\subsection{Single cell fabrication and indoor stability measurements}

OPV cells were initially prepared in a clean room environment using indium tin oxide (ITO) coated glass substrates $\left(R_{s}=16 \Omega /\right.$ square) that were cleaned using solvents, then treated in a UV-ozone reactor with oxygen plasma for 10 minutes. A layer of zinc oxide $(\mathrm{ZnO})$ film was deposited on ITO/glass substrate in a sputtering system at ambient temperature. Poly(3,4-

ethylenedioxythiophene)-poly(styrenesulfonate) (PEDOT:PSS) was spin coated on at 5000rpm for 30 seconds and baked on a hotplate at $120^{\circ} \mathrm{C}$, before moving the samples into a nitrogen glovebox. Active layer blends using $\mathrm{P} 3 \mathrm{HT}$ and $[6,6]$-phenyl- $\mathrm{C}_{61}$-butyric acid methyl ester $\left(\mathrm{C}_{61}-\mathrm{PCBM}\right)$, supplied by Nano-C Inc. USA, with weight ratios 1:0.8 were prepared and mixed with chlorobenzene solvent 
with a concentration of $30 \mathrm{mg} / \mathrm{mL}$. Prior to coating, the blend was allowed to dissolve for 24 hours on a hot plate stirrer and filtered using a $0.45 \mu \mathrm{m}$ PTFE filter. Samples were transferred into a nitrogen atmosphere glovebox $\left(\left[\mathrm{O}_{2}\right],\left[\mathrm{H}_{2} \mathrm{O}\right]<1 \mathrm{ppm}\right)$, where the active layer was applied by spin-casting from a $60^{\circ} \mathrm{C}$ solution (1500rpm for 60 seconds). The active layer was annealed at $140^{\circ} \mathrm{C}$ for 1 hour. Finally, thermal evaporation of the anode was performed through a shadow mask to define device area and consisted of $10 \mathrm{~nm}$ of molybdenum trioxide $\left(\mathrm{MoO}_{3}\right)$ and $100 \mathrm{~nm}$ of silver (Ag).

The LDS layer was dissolved in PMMA $(20 \mathrm{mg} / \mathrm{mL})$. The films were formed by doctor blading, with a final thickness measured at $\sim 1 \mu \mathrm{m}$. The doctor bladed layers were dried at room temperature for one hour. The LDS used for these tests was Tris(hexafluoroacetylacetonate) mono(1,10phenanthroline)europium(III) [sym.: Eu(hfac)(phen)], which was purchased from Lumtec, Taiwan and used as received. Overall, a schematic of the cell is shown in Figure 1(a) and an image of the LDS under UV excitation is shown in Figure 1(b). Absorption and transmission were measured with UVVis-NIR (UV-3600 SHIMADZU). Photoluminescence was measured with Fluoromax-4 spectrophotometer.

OPVs were checked for initial performance prior to lifetime testing using a Newport solar simulator with $100 \mathrm{mWcm}^{-2} \mathrm{AM} 1.5 \mathrm{G}$ output (calibrated using a silicon reference cell from RERA in the Netherlands) and a Source Measurement Unit (SMU) for taking current density-Voltage (J-V) measurements. For stability measurements, the cells were tested in accordance with ISOS-L-2 standards [14]. The cells were placed under the solar simulator for light soaking and were kept at open circuit in between measurements, with J-V measurements were made every 30 minutes for 550 hours. Whilst the temperature of the cells was not controlled, it was measured and stayed relatively constant during the testing at $40^{\circ} \mathrm{C} \pm 5^{\circ} \mathrm{C}$.

\subsection{Large area LDS printing for outdoor testing}

In order to apply the LDS coatings over larger areas for outdoor performance monitoring, a DEK 248 screen printer was used for printing the of the Eu(hfac)(phen):PMMA (abbreviated to 'Eu:PMMA') inks. For screen printable inks, the LDS layer was dissolved in PMMA $(10 \mathrm{mg} / \mathrm{mL})$ with $4 \%$ wt polyethylene glycol 400 (PEG 400) to act as a binder. The LDS coatings were printed onto a polyethylene terephthalate (PET) carrier substrate of thickness $=125 \mu \mathrm{m}$, supplied by DuPont Teijin Films, UK. Trials were performed to optimise the ink transfer onto the substrate by study the level of the snap- off gap and squeegee load that produced acceptable prints for the Eu:PMMA inks. After optimisation, it was discovered the optimal flood and print speed was $70 \mathrm{~mm} / \mathrm{min}$ and Squeegee load was $10 \mathrm{~kg}$. The PMMA inks were dried at $90^{\circ} \mathrm{C}$ for $3-5 \mathrm{~min}$ on a belt dryer. The LDS layers were printed into rectangular patterns with dimensions of $8.5 \times 10 \mathrm{~cm}$. As the film thickness of the screenprinted LDS layer was $5 \mu \mathrm{m}$, wave-guiding losses to the edge of the substrate were minimised and are estimated to contribute also to $<0.5 \%$ of optical losses. As the printed LDS were slightly thicker than the layers described in section 2.1, the concentration of LDS material was reduced to compensate. Measurements of the surface roughness of the printed LDS layer show the formed layer is very smooth $\left(R_{A}=3 n m\right)$. Figure 2(a) shows a photograph of the PET substrate before and after $2(b)$ coating of the LDS layer, with no obvious change in visible appearance. The photoluminescence from UV excitation, of the uncoated and coated LDS samples are shown in 7(c) and 7(d), respectively, confirming the uniformity of the LDS material dispersed in PMMA after screen printing. 


\subsection{Module (16-cell) fabrication and outdoor stability measurements}

Roll-to-roll (R2R) coated OPV modules were produced without UV-filter and used for the outdoor performance tests. The fabrication of these modules followed the literature reports with the exception that the barrier material employed did not include a UV-filter, as part of the 'free OPV program' at www.plasticphotovoltaics.org $[15,16]$. The devices had an ITO free structure of Carbon/PEDOT:PSS/ZnO/P3HT:PCBM/PEDOT:PSS/Carbon/PET-substrate (Figure S1 in Supporting Information). Outdoor performance monitoring of these type of R2R coated OPVs, which possess only a PET barrier layer is known to lead to rapid degradation in less than 3 months of outdoor operation [17]. Therefore to ensure long term stability, the modules were encapsulated in PDMS prior to testing. PDMS has been used in polycrystalline silicon PVs as an alternative to EVA and it only weakly affects UV light transmission from $400 \mathrm{~nm}$ to $280 \mathrm{~nm}$ [18] Therefore, the encapsulant does not significantly filter the UV components of incident sunlight, enabling the effectiveness of the LDS layer to be evaluated, whilst preventing oxygen and water ingress. A comparison of three configurations was undertaken, as shown in figure 2(e): (i) a control module without a UV filter, (ii) a module with a commercially available UV blocking layer from Solaronix SA (part number 49132) and (iii) a module with the Eu:PMMA LDS layer (printed on PET as described above). The Eu:PMMA LDS layer was attached to the top of the active area using a thin layer of PMDS to act as an adhesive [figure 2(e)].

Outdoor current-voltage (I-V) measurements of Roll-to-roll (R2R) coated OPV modules were performed for a period of 70 days during daylight hours (from 10 AM to 3 PM 6 times a day) starting from 28.12.2014 in Sede Boker (the Negev desert, Israel). This was conducted in accordance with ISOS-O-2 standards [14]. Global intensity of incident sunlight was measured with a calibrated thermopile pyranometer (Eppley PSP). During the measurements the modules had ambient temperatures measured by thermocouples. The stand was placed outdoor during the entire measurement period. It should be noted that the spectrum measured at noon time $\pm 2-3$ hours' of a cloudless day at Sede Boker (Lat. $30.8^{\circ} \mathrm{N}$, Lon. $34.8^{\circ} \mathrm{E}$, Alt. $475 \mathrm{~m}$ ) matches almost identically to the AM 1.5G spectrum [19], providing a vigorous test for the ability of the Eu:PMMA layer to filter UV light.

\section{Results and discussion}

\subsection{Photophysical characterisation}

In this work, LDS coatings were prepared by using Eu(hfac)(phen) dissolved in a PMMA host layer (abbreviated to 'Eu:PMMA'). The Eu:PMMA films were prepared and their photo-conversion properties were studied by measuring excitation and emission spectra and luminescent efficiency (Figure 3). Samples were prepared with $2 \%$ weight LDS in PMMA and applied to a glass sample using doctor blading, leading to a coated thickness of around $1 \mu \mathrm{m}$. Light from the LDS layer is emitted isotropically; therefore losses can occur away from the cell and through wave guiding perpendicular to the active layer. Wave guiding losses are minimised by ensuring the LDS layer is as thin as 
possible and it is estimated that these amount to less than $1 \%$ of losses occur as a result, when considering the samples sizes used for these tests.

The reasoning for using Eu(hfac)(phen) is shown well in Figure 3: its large Stokes shift prevents reabsorption of emitted light and good spectral breadth in the UV region limits UV light transmission into the active layer. For the Eu:PMMA sample, UV absorption occurred from 300nm up to around $415 \mathrm{~nm}$ with the peak absorption occurring at $\lambda_{\text {peak,abs }} \approx 360 \mathrm{~nm}$. A narrow luminesce is observed with a peak emission at $610 \mathrm{~nm}$, which is consistent with other europium phosphor complexes [20]. Overlaid on the data is the External Quantum Efficiency (EQE) of a P3HT:PCBM OPV and it is apparent that the LDS material has an the overlap in the emission spectrum with the EQE of P3HT:PCBM.

\subsection{Single cell performance and indoor stability results, conducted in accordance ISOS-L-2}

Single cell devices were fabricated and tested using the Eu;PMMA , LDS with a schematic of device architecture shown in figure $4(a)$. The $J-V$ characteristics of the representative P3HT:PCBM cells both with and without the Eu:PMMA LDS is shown in figure 4 (a). OPVs were prepared with $2 \%, 5 \%$ and $8 \%$ weight LDS in PMMA, with the thickness kept approximately kept constant. The LDS layers were applied to a glass surface using doctor blading as the sample sizes were relatively small $\left(4 \mathrm{~cm}^{2}\right)$. The key performance parameters including open-circuit voltage $\left(V_{o c}\right)$, short-circuit current density $\left(J_{s c}\right)$, fill factor (FF) and Power Conversion Efficiency (PCE) are summarized in table 1.

For the control devices, fabricated without an LDS, the average short-circuit current density $\left(J_{s c}\right)$ was measured at $10.01 \mathrm{~mA} / \mathrm{cm}^{2}$ and PCE at $2.79 \%$. For devices fabricated with the Eu:PMMA LDS, the optimum concentration was found at $2 \% \mathrm{wt}$, with the average $\left(J_{s c}\right)$ measured at 10.54 $\mathrm{mA} / \mathrm{cm}^{2}$ and a PCE of $3.04 \%$. It can be seen from Figure 4(b), the EQE of the OPVs with Eu:PMMA layer has been moderately improved over non-coated samples. In particular, at wavelengths between $310-400 \mathrm{~nm}$, the EQE has been enhanced by application of the layer. Therefore, the relative increase in $J_{s c}$ can be attributed to the UV down-conversion, leading to an increase in visible light into the OPV active region. The level of PCE increase is small and the primary reason for this is due to low proportion of UV light in the AM1.5G. Based on the Standard Reference Spectra are (ASTM G173-03), UV energy accounts for only $9.16 \%$ of light irradiation from the sun (AM1.5G). In addition, light is emitted isotopically from the LDS complex and light that possesses an angle of up to $38^{\circ}$ perpendicular to the OPV surface is likely emitted out of the PMMA host layer. Based on this emission escape cone calculation and the low waveguide losses due to the thinness of the LDS layer, there is only likely to be a maximum of $~ 6.9 \%$ relative increase in performance by application of an LDS layer onto an OPV cell, which indicates that the Eu:PMMA layer used in these devices is operating at around $57 \%$ of the maximum attainable enhancement, which could be improved by using materials with higher photoluminescent quantum yields. However, one of the principle advantages of the LDS layer is that it eliminates the need for a conventional UV filter. Figure 4(b) shows the EQE of the device with a commercial UV filter applied (Solaronix SA, part number 49132). It is evident that any light with a $\lambda<400 \mathrm{~nm}$ does not generate photocurrent, which leads to an overall drop in the solar cell performance. 
The effectiveness of the LDS for protecting OPVs against photo-degradation was tested by indoor light soaking using the AM1.5G spectrum in accordance with ISOS-L-2 standards. Lifetime stability tests were carried out for 550 hours for non-encapsulated devices with and without the Eu:PMMA LDS layer. Figure 5 shows how the key performance parameters of $\mathrm{V}_{\mathrm{OC}}, \mathrm{J}_{\mathrm{SC}}, \mathrm{FF}$ and PCE for the $\mathrm{P} 3 \mathrm{HT}$ :PCBM solar cells changed relative to their initial values during light soaking. As expected, the overall performance of devices without encapsulation decreased rapidly with trend matching closely an exponential decay, emphasising the need for encapsulation if devices are to be put to practical use $[21,22]$.

It is also evident that for devices fabricated with an LDS layer, the stability was much improved. For the control device, the time taken to reach 50\% of the original PCE $\left(T_{50 \%}\right)$, is 38 hours. This compares with $T_{50 \%}=322$ hours for the device with Eu:PMMA, leading to an overall improvement in half-life by $850 \%$. When considering the relative changes of each parameter for the devices, the main causes of the rapid decrease in $\mathrm{t}_{1 / 2}$ are due to reductions in $\mathrm{V}_{\mathrm{OC}}, \mathrm{FF}$ and $\mathrm{J}_{\mathrm{SC}}$ (with this order of precedence). The drop in $\mathrm{V}_{\mathrm{OC}}$ can be equated to a modification of the HOMO of P3HT, due to polymer doping [23]. It is known that upon exposure to oxygen and UV-light, a shift of the occupied states of about $0.3 \mathrm{eV}$ is observed, which correlates well to the measured drop in $\mathrm{V}_{\mathrm{OC}}$ $\left(\Delta \mathrm{V}_{\mathrm{OC}} \approx 0.27 \mathrm{~V}\right)$ in the control experiment. For the control device, no UV filtering is present, so this modification occurs rapidly after the commencement of the experiment ( $\mathrm{t} \sim 100$ hours). The fall in FF and $\mathrm{J}_{\mathrm{Sc}}$ follows the expected trend with other papers [21], showing a negative exponential drop from $\mathrm{t}=0$ hour, due to the chemical and physical degradation mechanisms of the photoactive PCBM:P3HT layer and electrode interfaces. $T$ is worth noting, the $V_{\text {oc }}$ and FF of OPV with such a device architecture may also degrade under UV-light due to generation of shunts in the $\mathrm{ZnO}$ hole blocking layer [15].

\subsection{Module (16-cell) performance and outdoor stability results, conducted in accordance ISOS-0-2}

Roll-to-roll (R2R) coated OPV modules prepared without UV-filter were used for evaluating the performance of the LDS layers in outdoor environments. Previous tests on these modules showed rapid degradation as the barrier layer possesses a relatively high Water Vapour Transmission Rate (WVTR) [24]. Therefore, the OPV module was entirely encapsulated in PDMS to limit the water and oxygen penetration into the active layer. PDMS was selected due to its high UV transparency, as shown in Figure $6(a)$, which shows the absorption cut-off of PDMS at around $\lambda \approx 298 \mathrm{~nm}$, which is beyond the spectral range of AM1.5G. To compare the effectiveness of the LDS layer, three samples of each configuration were prepared; three control samples with no UV filter, three modules with a laminated commercial UV filter (the transmission profile shown in Figure 6(a)) and three modules with the screen-printed Eu:PMMA filter attached (an image of the module illuminated with UV light is shown in Figure $6(b))$. All of the studied modules and the pyranometer were mounted on a fixed angle ( $30^{\circ}$ to horizontal) stand (FAS) (Fig. 6c).

Table 1 shows the averaged solar cell performance the OPV modules under AM1.5G irradiation, prior to outdoor stability testing. The best performing module is fabricated with the Eu:PMMA LDS layer, which shows the highest $\mathrm{J}_{\mathrm{SC}}$ and PCE, despite a lower $\mathrm{V}_{\mathrm{OC}}$ and FF. In Table SI- 1, the initial performance data (before application of the UV filters or PDMS encapsulation) for all modules are shown. These data indicate that the modules initially matched closely in performance. 
The EQE of the modules with and without LDS layers and with the commercial UV filter are shown in Figure SI-2. One of the main advantages of the OPV modules with the Eu:PMMA LDS layer becomes evident when comparing their performance to that for the modules with the commercial UV filter in table 1. By applying the UV filter, a substantial decrease in $\mathrm{J}_{\mathrm{SC}}$ and PCE is observed, indicating that a compromise in efficiency is normally needed to obtain the improved stability when using such a filter. The principle reason for this decrease is the high refractive index of the commercial UV filter; typically these are based on metal oxide films (with $n \sim 2.5$ ). Such as high refractive index leads to increased reflection of incident light and haze. In contrast, the variations in refractive indices of the layers used in the LDS-coated module are small, leading to limited normal incidence reflection. Therefore, by using an LDS layer as the UV filter, the performance drop is removed, whilst retaining the characteristic to filter out UV light.

The PCE of OPV modules operating outdoor have a complicated dependence on time during the day due to diurnal variation of the intensity and spectrum of sunlight and ambient temperature. This dependence is beyond the scope of the present paper and will be reported elsewhere. Here, for analysis of the OPV outdoor stability, we selected only one value of the module parameters (PCE, FF, $\mathrm{V}_{\mathrm{oc}}$ and $\mathrm{I}_{\mathrm{sc}}$ ) for every day of testing, which corresponded to the moment of maximum PCE (during a day). These values were normalized in the following manner; firstly, $J_{s c}$ values were adjusted to the standard irradiance value of $1000 \mathrm{~W} / \mathrm{m}^{2}$, by assuming a linear increase of $J_{s c}$ with light intensity [25]. $V_{o c}$ values were then adjusted to the standard temperature of $25^{\circ}$ assuming light decrease of the $O P V V_{o c}$ with temperature [25]. Then all values of PCE, FF, $V_{o c}$ and $I_{s c}$ were normalized to their initial values (results of the first out-door measurements in Sede Boker on 28.12.2014). Figure 7 (a-d) illustrates evolution of such normalized parameters for a representative module over a 70 day testing period in Sede Boker. Based on this data, it can be concluded that:

(1) PDMS modules (without any UV-filter or LDS layer) exhibited the fastest degradation of the PCE (Fig. 5a). The worst performing module after degradation was found to have $57 \%$ of the initial PCE value by the end measurement period. Meanwhile modules with UV filter and Eu:PMMA LDS layer showed considerably slower degradation during the long term test (their PCE decreased to only $75 \%$ of the initial values). The results show the Eu:PMMA improves the time for the modules to drop $75 \%\left(\mathrm{~T}_{75 \%}\right)$ of the original value by $51 \%$.

(2) During first 50 days of the experiment outdoor PCE degradation occurred mostly due to the degradation of FF (Fig. $5 c$ ) while $V_{o c}$ and $J_{s c}$ exhibited relatively stable behavior (Fig. 5 a, b). However, during the final exposure period (days 50-70) reduction of $\mathrm{V}_{\mathrm{oc}}$ and $\mathrm{J}_{\mathrm{sc}}$ was found to contribute to the PCE degradation as well.

There is a marked difference in the in the kinetics of the photovoltaic performance degradation of non-encapsulated samples (Fig. 5 and 8a) and encapsulated modules (fig. 7 and 8b). The encapsulated modules are observed to possess an initial "stable period," before degrading at a much quicker rate thereafter (see figure 8b), which is a trend observed in other OPV modules [19]. We can speculate that this trend originates from the gas permeation through the encapsulation layer and the initial stability is due to the "transition phase" in which steady state gas diffusion has not been reached, resulting in very small or no observable permeation and limited OPV performance 
fall-off. This is followed by a steady-state regime where diffusion has reached equilibrium, giving a constant flux of gas species permeating through the barrier films during this period oxygen or moisture can penetrate into the encapsulated sample or/and degradation of the encapsulation layer occurs.

\section{Conclusions}

The proof of concept of using LDS layers as UV filters for P3HT:PCBM OPVs has been demonstrated. Non-encapsulated single cell devices were fabricated and tested indoors. By applying the LDS layer, a $5 \%$ relative enhancement in photocurrent and an $850 \%$ enhancement in the half-life $\left(T_{50 \%}\right)$ were observed using indoor light testing protocols, indicating improvement in both performance and lifetime. Encapsulated modules based on 16-cell R2R-coated OPVs with the LDS layer were also tested under indoor test conditions and showed a $6.5 \%$ relative enhancement in photocurrent, when compared to a control device. More significantly, modules prepared with a commercial UV filters showed a substantial decrease in photocurrent (-17.5\%), indicating that LDS layers could be used as alternative UV filters without compromise to device efficiency. The OPV modules were tested for outdoor stability over a 70 day period in the Negev desert, Israel and the modules made with the LDS filter are shown to match the performance of those using the commercial UV filter and outperform the modules with no filter applied. The work indicates that the LDS layer can act as a UV filter for substantial improvement of the OPV operational stability, without a loss in photocurrent as witnessed with the commercial filter.

\section{Acknowledgements}

JK and EK would like to acknowledge the support of support of the European Commission's StableNextSol COST Action MP1307. JK would like to thank Sêr Cymru national research network in Advanced Engineering and Materials. In addition, JK and DG would like to thank the Wales Ireland Network for Innovative Photovoltaic Technologies (WIN-IPT) project, funded through Interreg IVA. Dr Ziqian Ding is thanked for the device fabrication and experimental testing.

\section{References}

1. C.C. Chen, W-H. Chang, K. Yoshimura, K. Ohya, J. You, J. Gao, Z. Hong, Y. Yang, Adv. Materials, 2014, 26, 32, 5670-5677.

2. H. Cao, W. He, Y. Mao, X. Lin, K. Ishikawa, J.H. Dickerson, W.P. Hess, J. of Power Sources, 2014, 264, 168-183.

3. N. Grossiord, J. M. Kroon, R. Andriessen, P.W.M. Blom, Org. Electronics, 2012, 13, 3, 432-456.

4. J. Abad, N. Espinosa, R. García-Valverde, J. Colchero, A. Urbina, Sol. Energy Mat. and Solar Cells, 2011, 95, 5, 1326-1332. 
5. M.O. Reese, A. M. Nardes, B.L. Rupert, R. E. Larsen, D.C. Olson, M.T. Lloyd, S.E. Shaheen, D.S. Ginley, G. Rumbles, N. Kopidakis, Adv. Func. Materials, 2010, 20, 20, 3476-3483

6. M.S. Ryu, H. Cha, J. Jang, Sol. Energy Materials and Solar Cells, 2010, 94, 2, 152-156.

7. D. Alonso-Alvarez, E. Klampaftis, D. Ross, B. S. Richards, Photovoltaics, IEEE Journal of , 2014, 4, 6,1532-1537.

8. S.D. Hodgson, S. Jones, P. J. Holliman, S. L. Rugen-Hankey, V. Barrioz, S. J. C. Irvinem Materials Letters, 2014, 130, 120-122.

9. M. Zahedifar, Z. Chamanzadeh, SM Hosseinpoor Mashkani, J. Luminescence, 2013, 135, 66-73.

10. W. Xu, H. Song, D. Yan, H. Zhu, Y. Wang, S. Xu, X. Bai, B. Dong, Y. Liu, J. Mat. Chem., 2011, 21, 33,12331-12336.

11. M. Prosa, A. Sagnella, T. Posati, M. Tessarolo, M. Bolognesi, S. Cavallini, S. Toffanin et al., RSC Advances, 2014, 84, 44815-44822.

12. L. H. Slooff, R. Kinderman, A. R. Burgers, N. J. Bakker, J. A. M. Van Roosmalen, A. Büchtemann, R. Danz, M. Schleusener. J. Solar Energy Engineering, 2007, 129, 3, 272-276.

13. S. Engmann, M. Machalett, V. Turkovic, R. Rösch, E. Rädlein, G. Gobsch, H. Hoppe,. Journal of Applied Physics, 2012, 112(3), 034517.

14. M. O. Reese, S. A. Gevorgyan, et al, Sol. Energy Mater. Sol. Cells. 2011, 95, 1253.

15. F.C. Krebs, M. Hösel, M. Corazza, B. Roth, M.V. Madsen, S.A. Gevorgyan, R.R. Søndergaard, D. Karg, M. Jørgensen, Energy Technology, 2013, 1, no. 7, 378-381.

16. G. A. dos Reis Benatto, B. Roth, M. V. Madsen, M. Hösel, R. R. Søndergaard, M. Jørgensen, F. C. Krebs, Adv. Energy Mater., 2014, 1400732

17. A. Manor, E. A. Katz, T. Tromholt, F. C. Krebs, Adv. Energy Materials, 2011, 1, 5, 836-843

18. O. Hasan, A.F.M. Arif, Sol. Energy Materials and Solar Cells, 2014, 122, 75-87.

19. E.A. Katz, S. Gevorgyan, M.S. Orynbayev, F.C. Krebs, The European Physical Journal Applied Physics, 2006, 36(03), 307-311

20. A. O'Riordan, E. O'Connor, S. Moynihan, X. Llinares, R. Van Deun, P. Fias, P. Nockemann, K. Binnemans, G. Redmond, Thin Solid Films, 2005, 491, 1, 264-269

21. C.H. Peters, T. Sachs-Quintana, J.P. Kastrop, S. Beaupré, M. Leclerc, M. D. McGehee, Adv. Energy Materials, 2011, 1, 4, 491-494.

22. H. Waters, N. Bristow, O. Moudam, S. Chang, C. Su, W. Wu, U. Jeng, M. Horie, J. Kettle, Org. Electronics, 2014, 15, 10, 2433-2438.

23. H. Hintz, H-J. Egelhaaf, H. Peisert, T. Chassé, Polymer Degradation and Stability, 2010, 95, 5, 818825. 
24. N. Bristow, J. Kettle, J. Renewable and Sustainable Energy, 2015, 7, 1,013111.

25. T. Tromholt, E.A. Katz, B. Hirsch, A. Vossier, F.C. Krebs, Appl. Phys. Lett., 2010, 96, 073501.

\begin{tabular}{|l|l|l|l|l|l|l|}
\hline Sample & PCE (\%) & $\mathrm{V}_{\text {oc }}(\mathrm{V})$ & $\mathrm{I}_{\text {sc }}(\mathrm{mA})$ & $\mathrm{FF}(\%)$ & $\begin{array}{l}\text { Relative } \\
\text { Isc change }\end{array}$ & $\begin{array}{l}\text { Relative PCE } \\
\text { change }\end{array}$ \\
\hline \multicolumn{5}{|l|}{ Single cell (For indoor testing using ISOS-I-2) } \\
\hline Control & 2.79 & 0.598 & 10.01 & 46.6 & & \\
\hline 2\% Eu:PMMA & 3.04 & 0.604 & 10.54 & 47.5 & +5.1 & +8.4 \\
\hline 5\% Eu:PMMA & 2.82 & 0.607 & 9.84 & 47.2 & -1.7 & +1.1 \\
\hline 8\% Eu:PMMA & 2.31 & 0.604 & 8.24 & 46.4 & -16.7 & -16.8 \\
\hline 16-cell Module (For outdoor testing using ISOS-O-2) & & & \\
\hline Control & 2.39 & 12.13 & 13.68 & 43.37 & & \\
\hline UV filter & 1.97 & 11.94 & 10.59 & 46.77 & -17.5 & $-22.8 \%$ \\
\hline Eu:PMMA & 2.44 & 11.70 & 14.62 & 42.97 & +6.5 & $+2.1 \%$ \\
\hline
\end{tabular}

Table 1: Performance of the OPV devices, measured under AM1.5G illumination. The single cells were used to optimise the Eu:PMMA concentration and an optimum of $2 \%$ wt in solution was found. The 16-cell (module) performance was measured and benchmarked against a control sample (no UV filter) and a module fitted with a commercial UV filter 


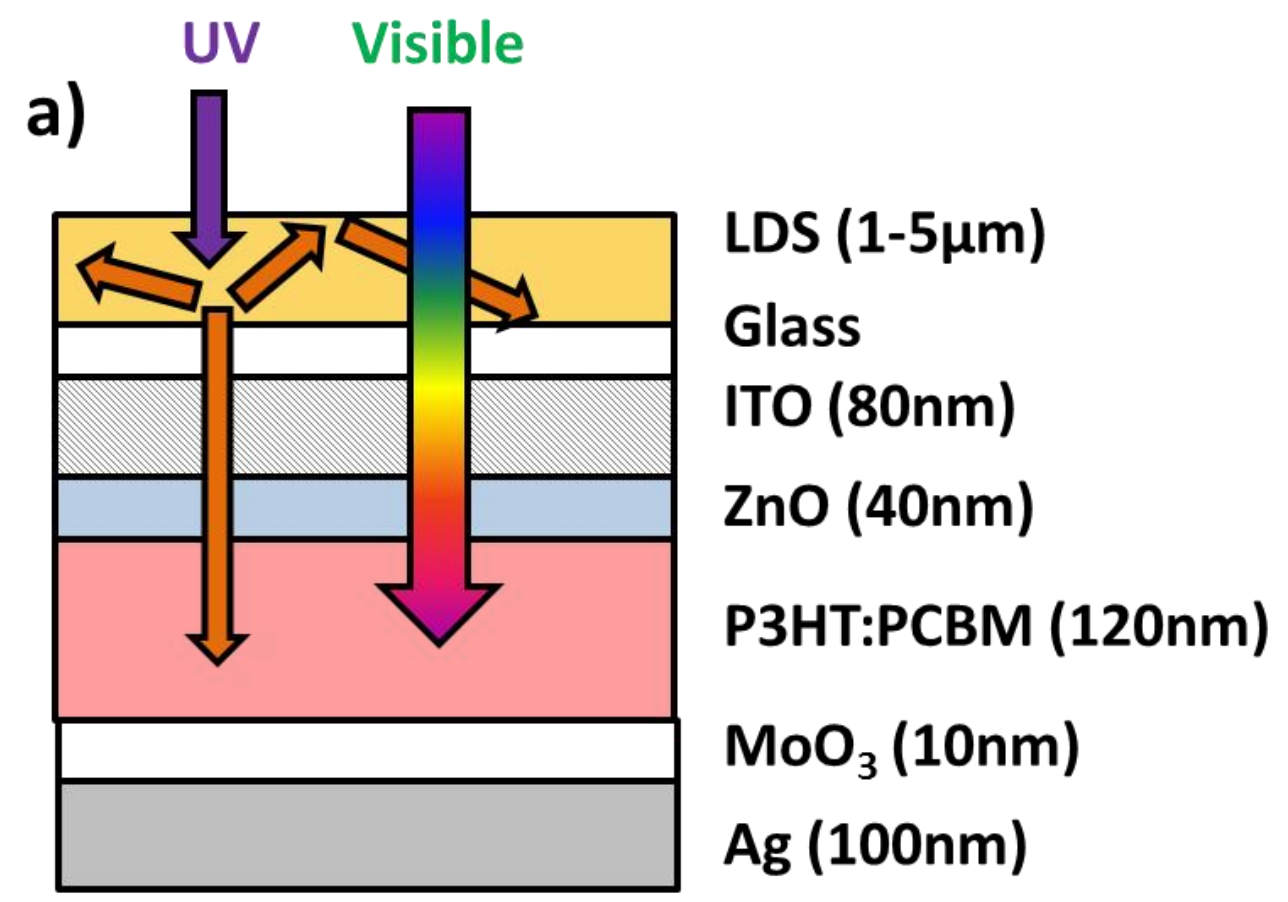

b)

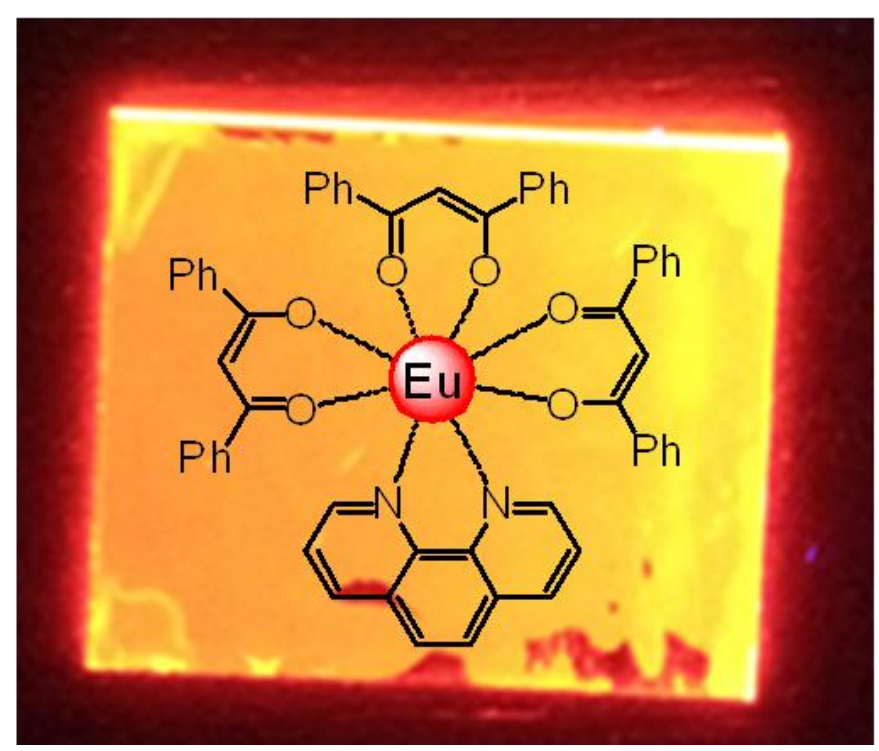

Figure 1 a) Schematic of the device used for single cell and 16-cell OPVs, with the LDS applied to the top surface and b) the Europium complex and photoluminescent response from above using UV excitation 


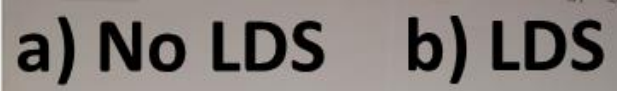
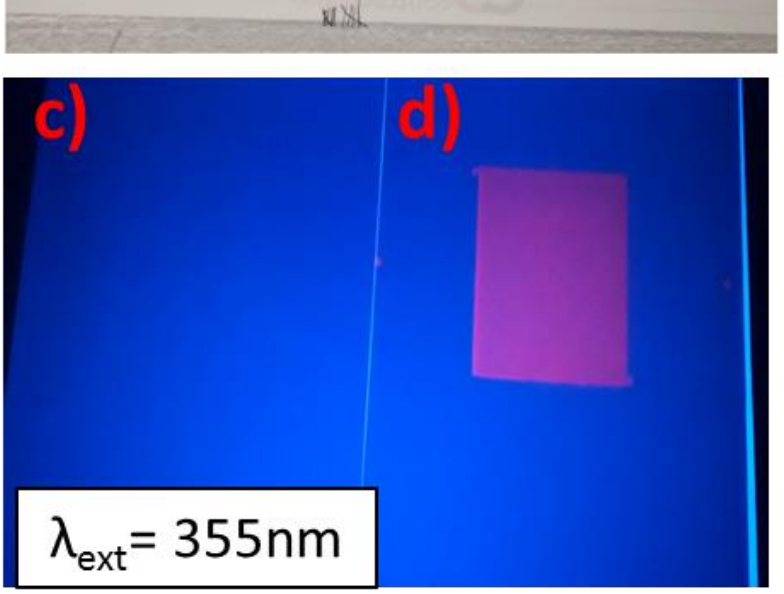

\section{e)}
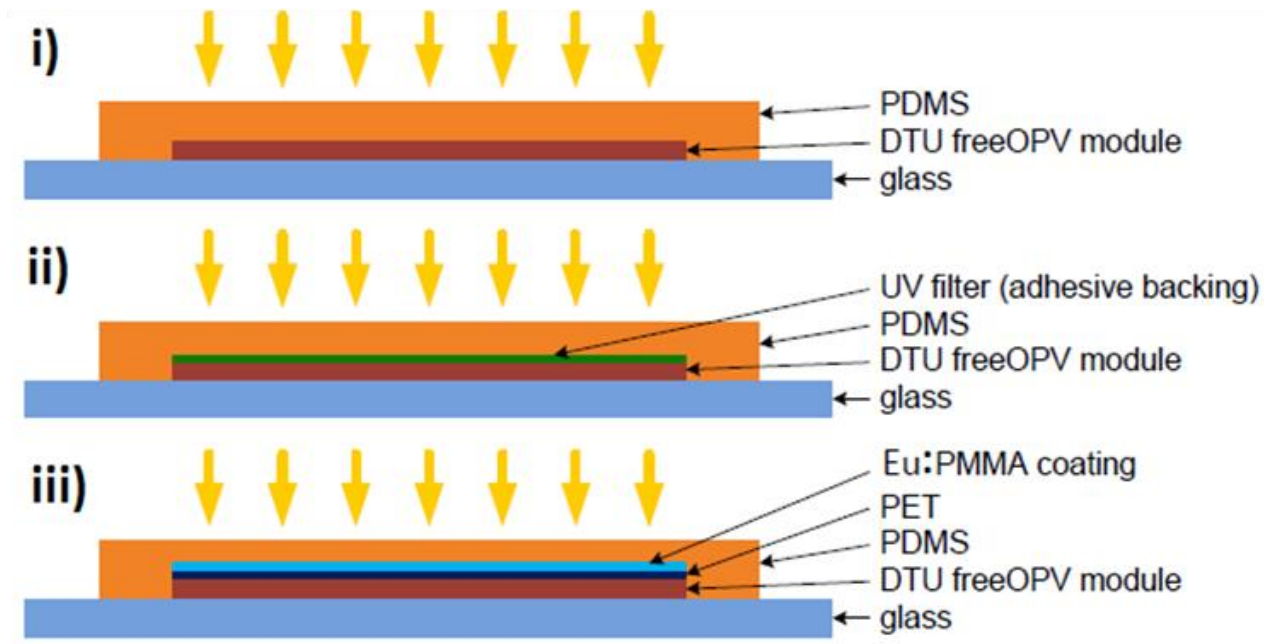

Figure 2: Roll-to-roll (R2R) coated OPV modules. Photograph of the PET substrate (a) without and (b) with the Eu:PMMA LDS printed, with photolum inescense response from UV excitation shown also for substrates (c) without and (d) with the Eu:PMMA LDS layers. The rectangular LDS coated area was cut and attached the OPV module using an optical adhesive layer (e) and encapsulated in PDMS. All modules were mounted onto a rigid glass substrate before outdoor testing. 


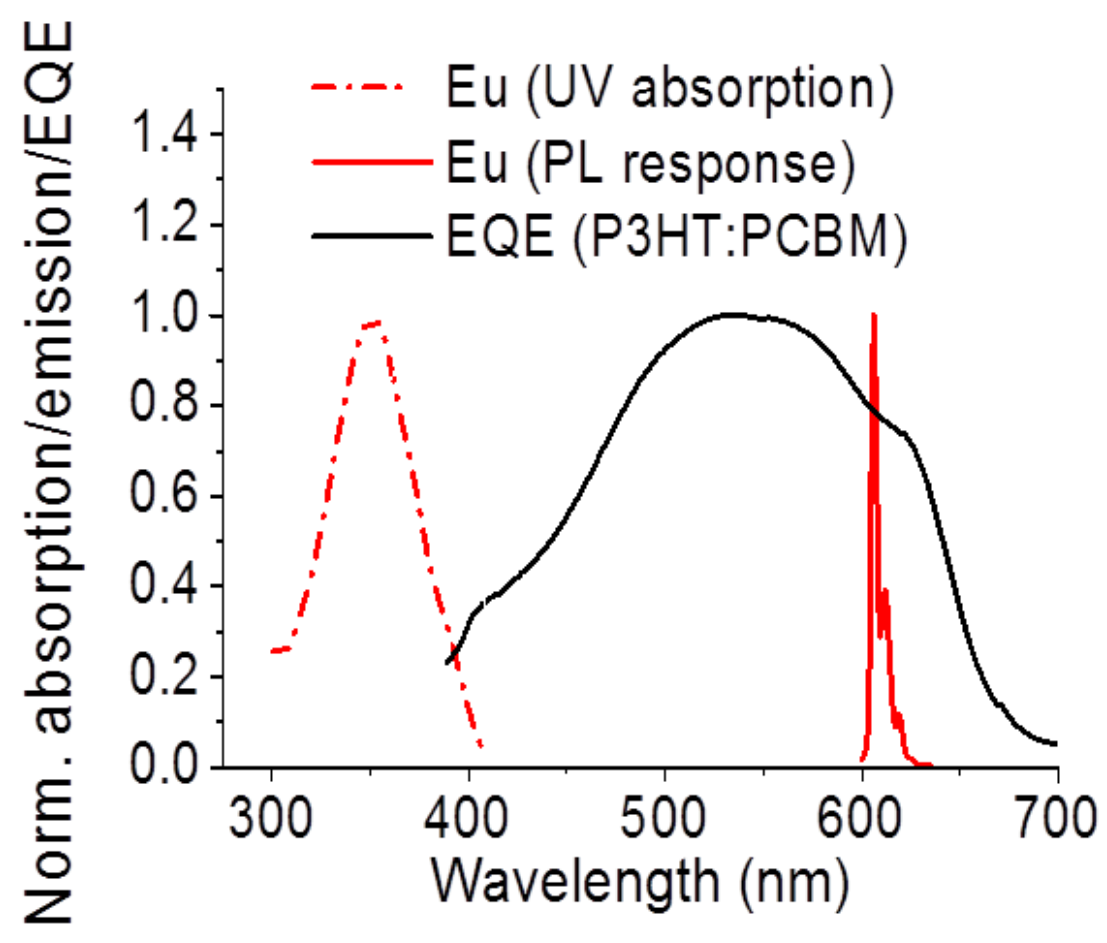

Figure 3: Relative absorption and photoluminescence $(P L)$ emission $\left(\lambda_{\text {exc }}=355 n m\right)$ of the Eu:PMMA layer. The PL response shows good overlap with the External Quantum Efficiency (EQE) of P3HT:PCBM solar cell (also shown) 

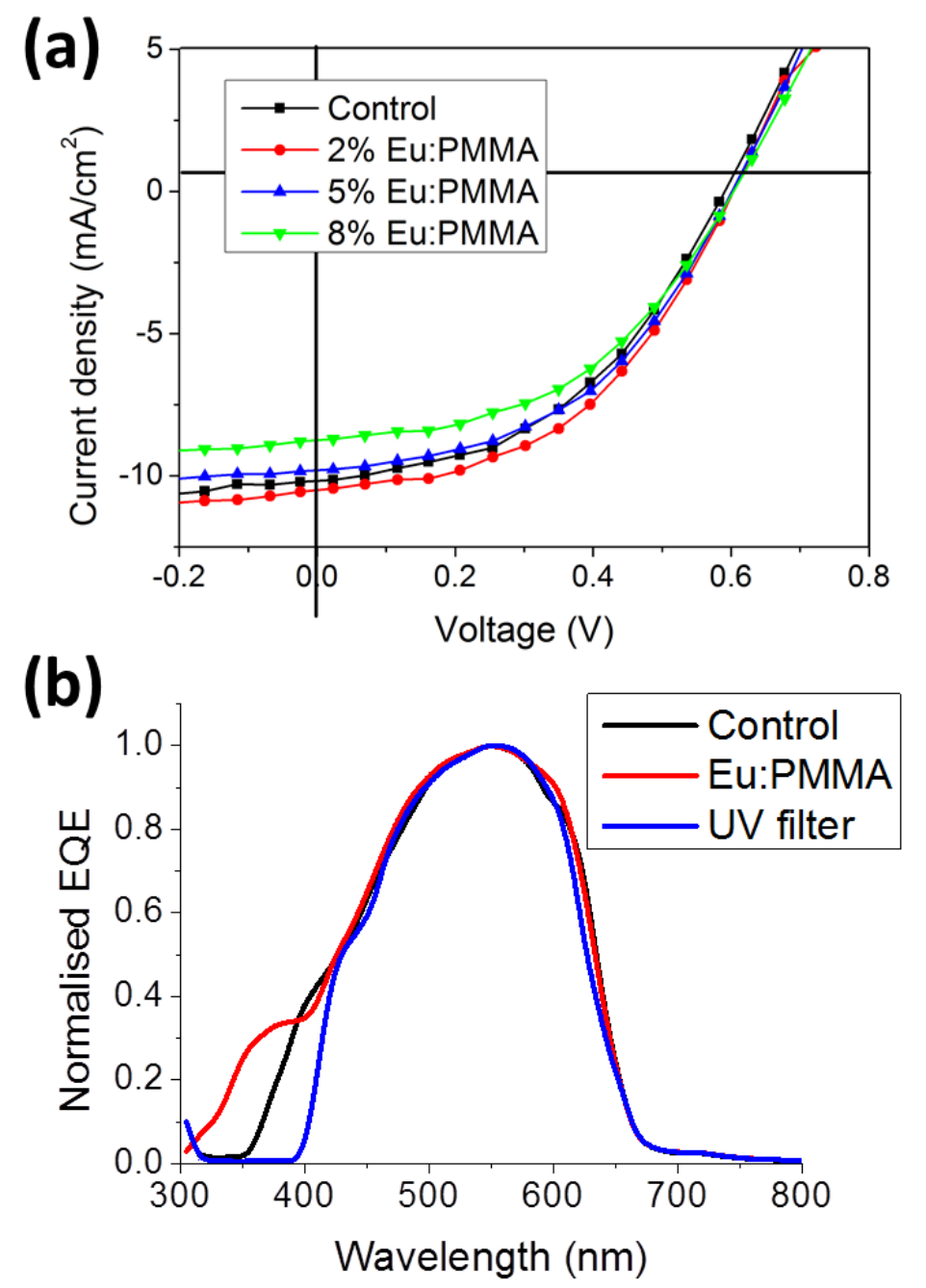

Figure 4(a) The measured current-voltage characteristics under AM1.5G of the representative P3HT:PCBM cells without LDS and with EU:PMMA (2\%, 5\% and $8 \%$ ) and (b) the External Quantum efficiency (EQE) of the cells without LDS and with EU:PMMA (2\%) and also the performance with a commercially available UV filter from Solaronix SA (part no. 49132) 
(a)

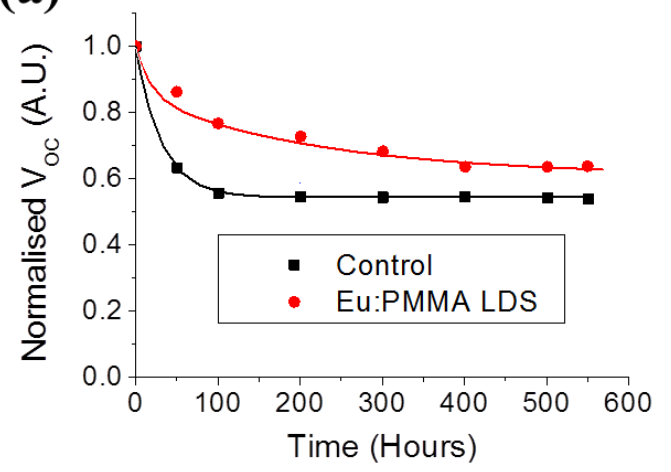

(c)

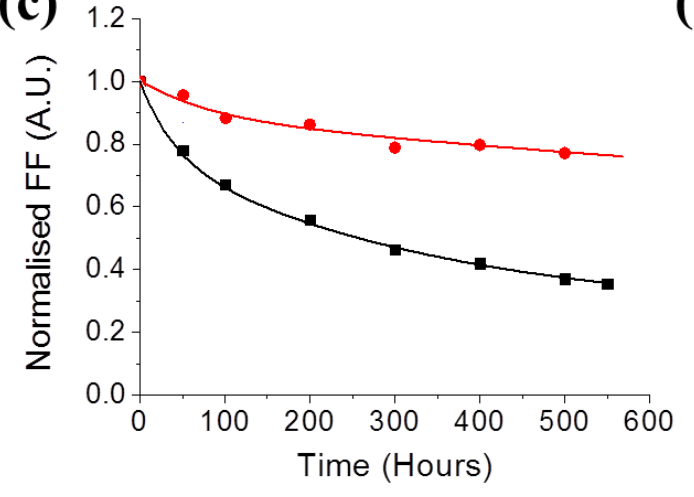

(b)

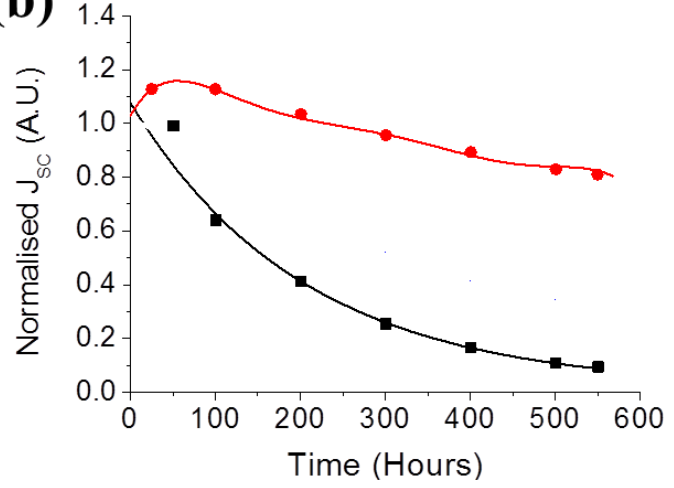

(d)

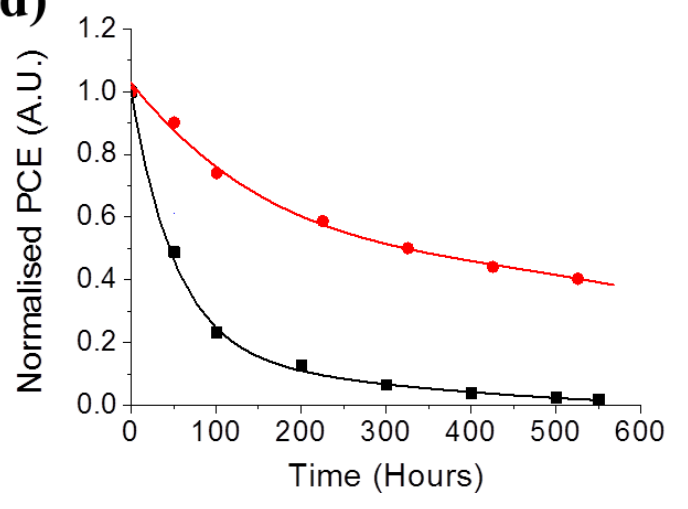

Figure 5. Evolution of the principle photovoltaic parameters $\left[(a) V_{O c},(b) I_{s c},(c) F F\right.$ and (d) PCE)] of the representative P3HT: PCBM cells with and without LDS under long term illumination at AM 1.5G condition $\left(100 \mathrm{~mW} / \mathrm{cm}^{2}\right)$ 

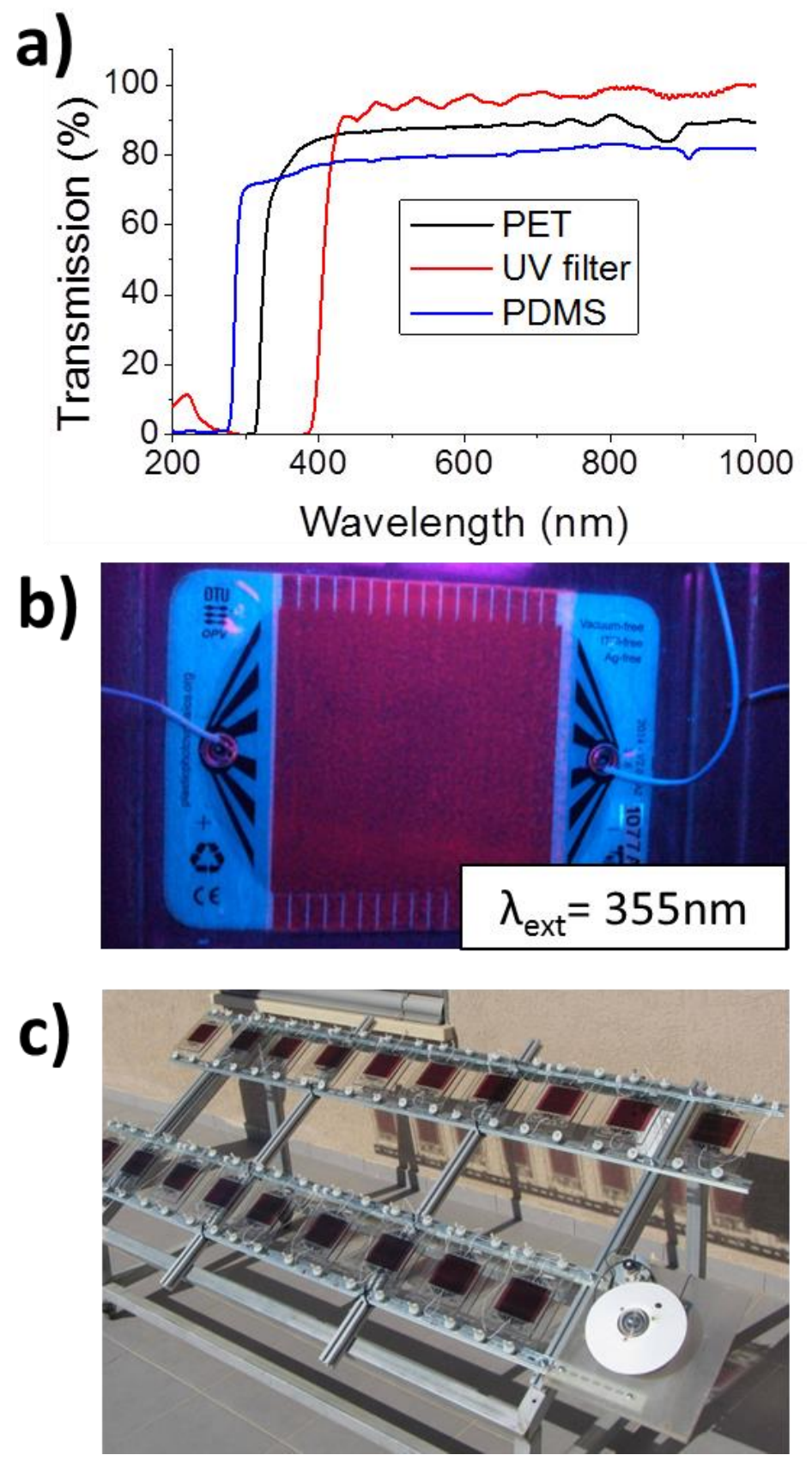

Figure 6: (a) Transmission of light through the PET, PDMS encapsulation layers and through the commercial UV filter b) image of the OPV module with LDS filter under UV excitation and (c) image of experimental setup at the Jacob Blaustein Institutes for Desert Research, which is part of the BenGurion University, Negev desert, Israel 

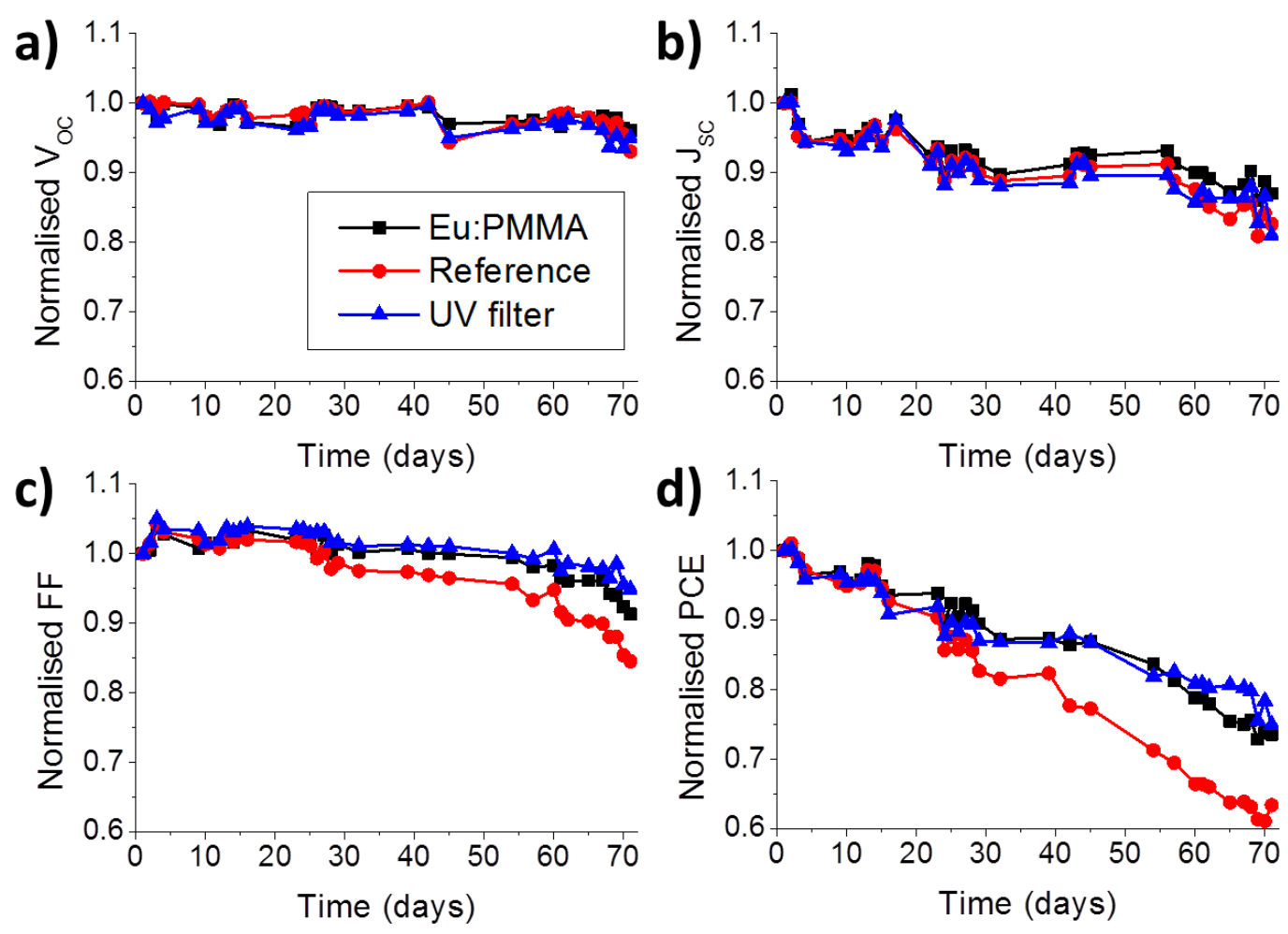

Figure 7: Evolution of principle photovoltaic parameters ((a) Voc, (b) Isc, (c) FF and (d) PCE) of the representative module over a 70 day period of outdoor solar exposure in the Negev desert for the i) control modules and ii) those with a commercial UV filter and iii) the Eu:PMMA LDS filter. Performance parameters were averaged across data from three modules. 


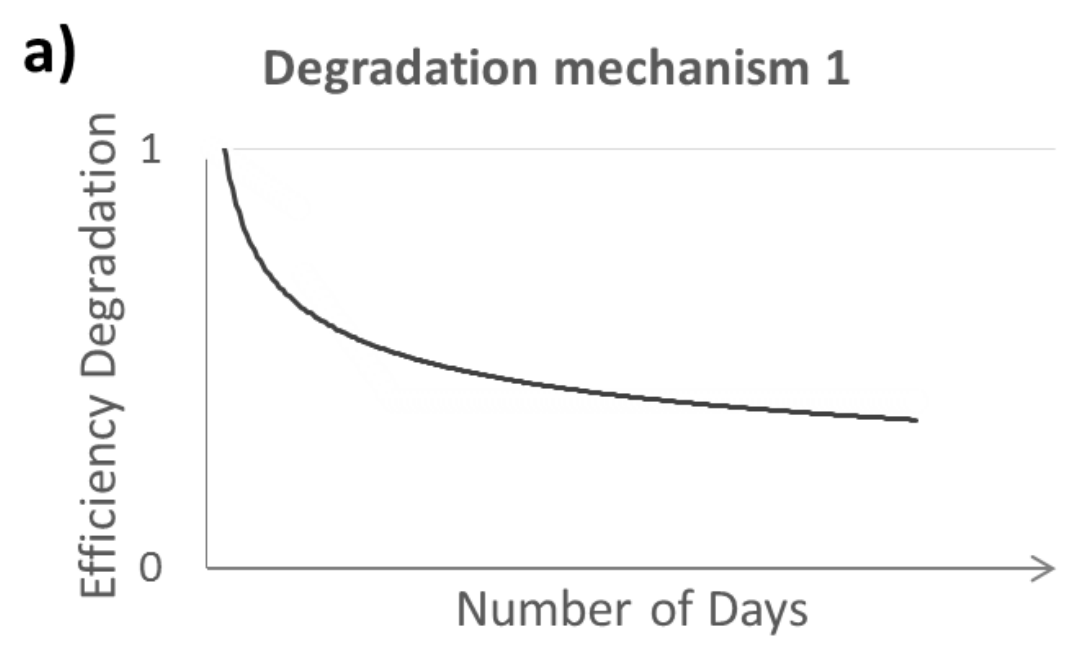

b)

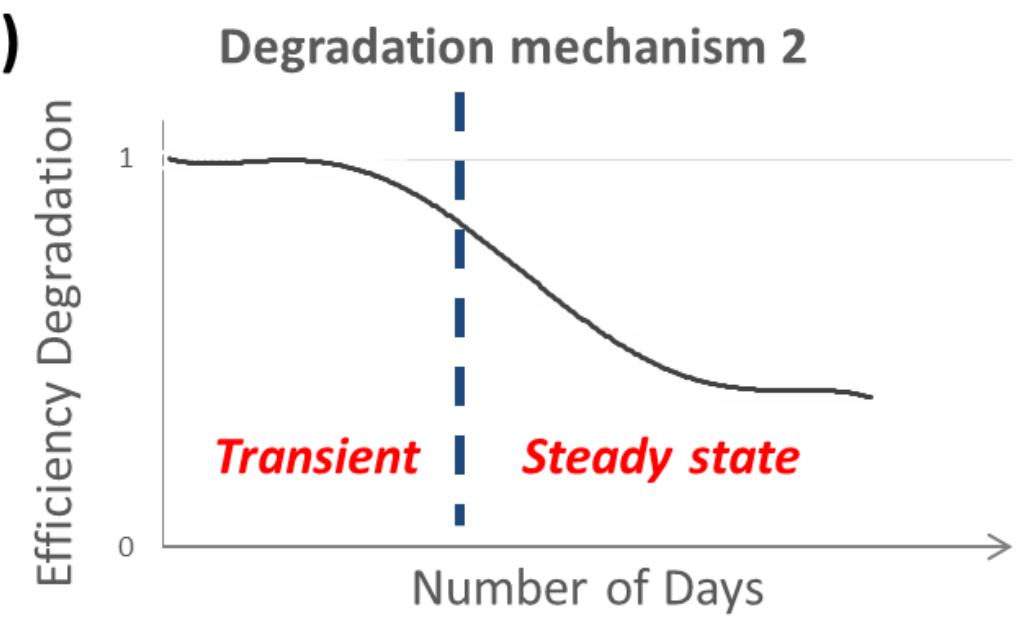

Figure 8: Degradation kinetics for non-encapsulated (a) and encapsulated OPV modules (b). 